\title{
Training of Basic Life Support Among Lay Undergraduates: Development and Implementation of an Evidence-Based Protocol
}

This article was published in the following Dove Press journal: Risk Management and Healthcare Policy

\section{Chun-yan Xie \\ Shu-lei Jia \\ Chao-zhu $\mathrm{He}$}

School of Nursing, Nanchang University, Nanchang 330006, People's Republic of China
Correspondence: Shu-lei Jia

Tel +86-1527-096-9394

Email ncdx0305@I63.com
Background: Cardiopulmonary resuscitation (CPR) is an important method to improve the prognosis of patients with prehospital cardiac arrest (CA). Basic life support (BLS) is the first step in CPR and is usually performed by the first witness. However, the general population has poor BLS skills due to the lack of efficient and practical training strategy. Several training initiatives could be used to improve this situation, and the challenge is to find the most efficient one in detail according to the actual setting. Repeated and effective BLS training increase bystander's confidence and willingness to perform BLS. Evidencebased instructional design is essential to improve the training of lay providers and ultimately improve resuscitation performance and patient outcomes.

Objective: 1) To develop an evidence-based BLS training protocol for lay undergraduates; 2) to implement the protocol and 3) to evaluate the process of implementation.

Methods: Nine databases were searched to synthesize the best evidence. A protocol was formed by ranking evidence and considering university setting and students' preferences. We implemented this training protocol and evaluated its effects.

Results: We achieved the three aims above. A total of 120 lay undergraduates received BLS training and retraining within 3 months. The students and teaching staff were satisfied with the training protocol and effect. The BLS training process was more clearly defined. The role of teaching assistants and the strategies to sustain training quality was proven to be crucial to the project's success.

Conclusion: The development and implementation of an evidence-based protocol could elevate undergraduates' BLS skill and confidence.

Keywords: training, basic life support, evidence-based, cardiopulmonary resuscitation

\section{Introduction}

Cardiopulmonary resuscitation (CPR) is one of the simple and effective ways to treat cardiac arrest (CA) due to a variety of causes. ${ }^{1}$ For critically ill patients, especially those with cardiac arrest or respiratory arrest due to heart attack, heat stroke, electric shock and drowning, the best rescue time is within 4-6 mins after the onset; otherwise, it may cause physiological death. ${ }^{2}$ Basic life support (BLS), also known as onsite first aid, refers to the professionals or laymen rescue patients by hand. The goal is to ensure the oxygen supply of the heart, brain and other vital organs. ${ }^{3}$ Various acute and critical diseases, disasters, and accidents often occur outside the hospital. The first to arrive at the scene are often laymen, so it is unrealistic and unscientific to rely on the arrival of emergency personnel for on-site treatment. ${ }^{4}$ Public on-site first aid refers 
to the activities of laymen to provide on-site treatment to patients with sudden emergencies, also known as firstwitness first aid. Several studies have documented the value of bystanders performing BLS on cardiac arrest, proving that most patients can benefit from effective BLS for laymen. ${ }^{5}$ First-aid organizations, such as the American Heart Association ${ }^{6}$ and the Chinese Research Hospital Association Cardiopulmonary Resuscitation Committee, ${ }^{7}$ recognize the use of BLS by laypersons as an important prehospital intervention. The bystander BLS, together with the social professional emergency system and the hospital ICU emergency service, set up a fast and convenient pathway to save lives and played an important role in emergency treatment.

An effective BLS popularization model has been established in developed countries, with a penetration rate of more than $30 \%$, and is protected by law. ${ }^{8}$ In contrast, the penetration rate of BLS in China is less than $1 \%,{ }^{9}$ and there is no standardized and unified public emergency training system. Although some institutions have conducted small-scale, temporary, inefficient, unsustainable, purely theoretical and unsatisfactory training activities, in most cases, due to some subjective and objective factors, coupled with a lack of effective training programs, the frequency of execution of laymen's BLS is still disappointing. ${ }^{10}$ The quality of college students' BLS skills training and their teaching strategies should be the focus of college education. ${ }^{11}$ College students, as the high-quality reserve force of society, are serving the masses, so first-aid skills are necessary to promote their all-round development, as well as a trend of international education reform and development.

The evidence-based approach is a process of earning the best evidence carefully, clearly, and wisely, and combining scientific research, practical experience, actual environment, and the preferences of beneficiaries to develop best practices. ${ }^{12}$ By establishing an evidencebased team, we integrated the best evidence retrieved into existing training practices in a process-oriented manner and formulated and applied BLS training programs for non-medical college students to improve their knowledge and skills in participating in first aid. A powerful radiating effect is gradually formed in society, accelerating the promotion of first-aid knowledge, and ultimately improving the level of public selfrescue and mutual rescue.

\section{Methods}

\section{Acquiring Evidence}

Nine databases, including the Cochrane Library, Joanna Briggs Institute (JBI) Evidence Health International Corporation Center Library, PubMed, Embase, Scopus, CINAHL, CBM, CNKI, and VIP were searched from set-up until June 2019 with terms of BLS/first aid, training/education/ cultivation/popularity, undergraduate/college/university. Two reviewers independently screened literature, extracted data, and cross-checked evidence. The third-party judged when conflicts emerged. Firstly, we read titles to remove unrelated documents. Then, we read abstracts and full texts to determine whether to adopt them. Six hundred twenty-four studies were found after initial checkout, and 7 of them were fitted according to the correlation of subjects and the degree of evidence. These were $2015^{6}$ and $2017^{13}$ American Heart Association Guideline Updates for Cardiopulmonary Resuscitation and Emergency Cardiovascular Care (level I), 2015 $5^{14}$ and $2017^{15}$ International Consensus (level I), 2016 Chinese Consensus ${ }^{7}$ (level IV), Systematic evaluation of training effect of highfidelity simulator (HFS) ${ }^{16}$ (level II), Simulation in resuscitation teaching and training: an evidence-based practice review $^{17}$ (level III) (Table 1).

\section{What the Evidence Says}

Several studies have proven the benefits of BLS training for lay undergraduates, but until recently, less attention has been paid to the quality, psychological factors of BLS training routine, and planned protocol in undergraduates. ${ }^{17}$ The factors that determine survival chance include early recognition of CA outbreak, first eyewitness performing

Table I Best Practice Recommendation Suggestions for Lay Undergraduates' BLS Training

\begin{tabular}{|l|l|}
\hline Content of Evidence & $\begin{array}{l}\text { Recommended } \\
\text { Level }\end{array}$ \\
\hline $\begin{array}{l}\text { Provide pre-class preparation of team } \\
\text { meeting }\end{array}$ & Recommendation A \\
\hline $\begin{array}{l}\text { Adjust teaching strategy according to pilot } \\
\text { training and assessment }\end{array}$ & Recommendation B \\
\hline Adopt CPR corrective feedback devices & Recommendation A \\
\hline $\begin{array}{l}\text { Chest compress only for non-medical } \\
\text { students, full CPR training for medical } \\
\text { students }\end{array}$ & Recommendation A \\
\hline Retrain in 3 months & Recommendation A \\
\hline
\end{tabular}


continuing compression-only BLS in time, acquiring automated external defibrillator (AED), and a local efficient and professional emergency medical service system (EMSS). ${ }^{14,15}$ Many studies have documented that the most common errors of resuscitation are inadequate compression rate and depth, both of which may reduce the patients' survival. 8,13,18,19 A systematic review reported that the high-fidelity simulator was positive and effective, superior to the traditional teaching methods. ${ }^{16}$ Therefore, using CPR feedback devices, especially corrective feedback, rather than reminder only, can guarantee the quality of compression. The effect of self-study by videos and computers combined with practice on a BLS model is equal to traditional teacher-centered lectures. ${ }^{6}$ Furthermore, pre-class activities could promote BLS study. Although people who have never studied BLS could be a rescuer by the guidance of phone as well, training can help boost their confidence. Only chest compression training is recommended for laymen. Multiple studies have proven the effectiveness and necessity of maintaining BLS training. In order to maintain BLS technique, retraining is desired in less than 2 years. ${ }^{6}$ After 3 months of observation and evaluation, the 24 mins DVD-based teaching combined with BLS self-training has the same effect as the 6 hrs BLS course. ${ }^{20}$ Many researchers have found that higher education of trainees guaranteed the effect of BLS teaching. 9,21,22 We also searched the literature to assess the challenge, which was a feedback loop focused on continuous quality improvement that can help the system improve as well as identify needs for targeted learning and performance. $^{6}$ To meet these challenges, several studies conducted BLS trainings for lay providers to find the best training formulation in Chinese scenarios. ${ }^{7,9,23}$ Our project was designed to address these challenges in implementing evidence-based change at one university in China.

\section{Analysis of Existing Problems}

According to the best evidence practice, the research team analyzed the current training practices and the reasons for the gap between the best evidence from four aspects to find potential obstacles and provide a basis for formulating a practical intervention plan. We used fishbone diagrams to analyze the factors influencing the popularity of BSL among lay undergraduates (Figure 1).

\section{Challenges and Facilitators}

Throughout the project, we encountered several challenges and facilitators of implementing BLS training. Getting support from the hospital emergency team was the first challenge to overcome. Emergency nurses and doctors did not consider it necessary to involve lay rescuers in prehospital first aid because they are not professionals and have not received professional training. Most of the time, laypeople are the first to arrive at $\mathrm{CA}$ site, and they often feel stressed, which may result in the incorrect performance of BLS and interference with the activities of ambulance personnel. They usually removed victims too quickly and took them to hospital in their vehicles. Emergency staffs believe that if a layperson is overly involved in BLS with their lack of professional skills, it will easily cause time waste, hamper emergency services,

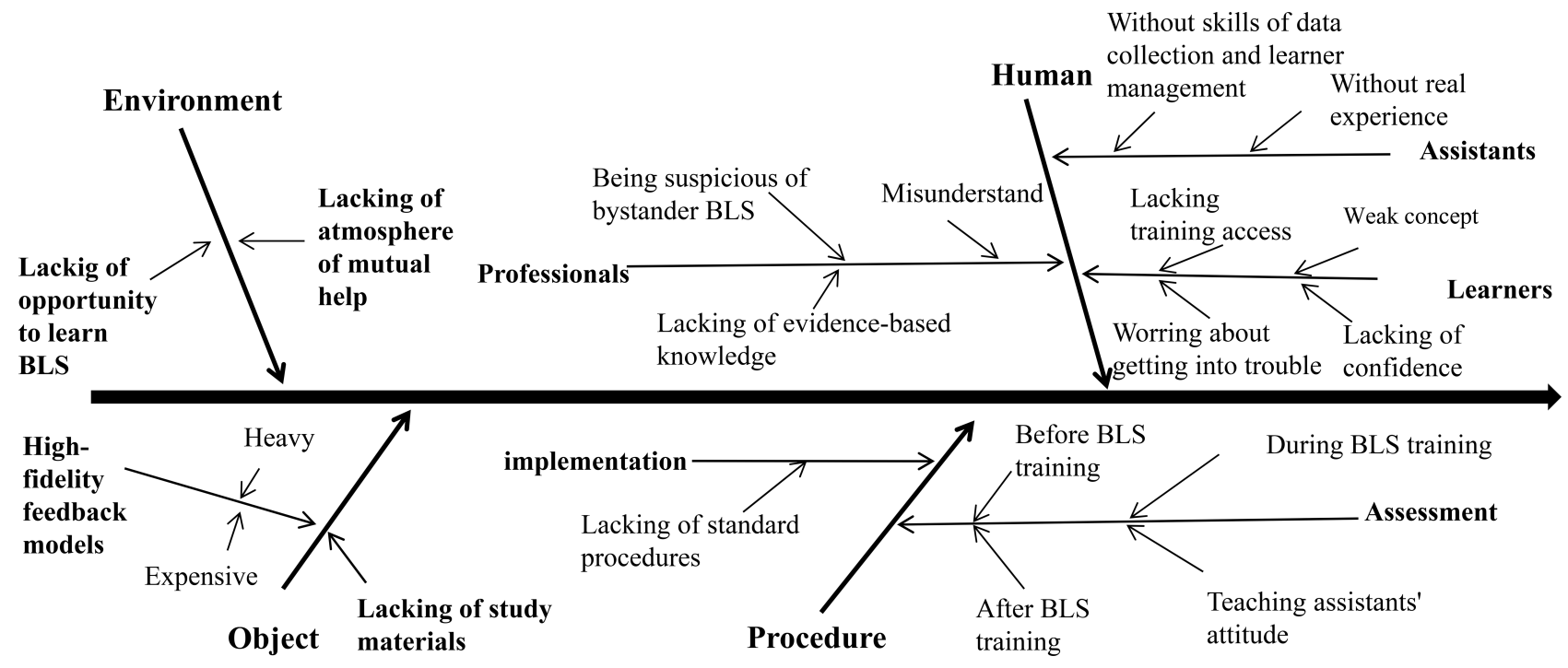

Figure I Fishbone diagram. 
and cause secondary harm to CA. However, their involvement was regarded as necessary and useful to alert the emergency services, especially for the rural and remote areas.

The undergraduate nursing volunteers were designated to attend the project as teaching assistants because peer education was regarded as a circulation of learning and teaching among similar background students. Originally, it was thought that using this teaching method was convenient, but this was not the case. Volunteers' unsure of themselves regarding never taking part in real BLS, lacking medical experience, and data collection was finally addressed in the simulation. In addition, some studies $^{24-27}$ showed that nearly half of the laypersons who were trained in BLS believed they knew the procedure but could not perform BLS in real situations, which suggested that the confidence of performing BLS was not satisfactory. Therefore, psychological preparation for both assistants and learners was a priority.

Procedure challenges of data collection proved more time-consuming than originally planned. Since the assistants were university students, they were easily disturbed by campus activities and personal affairs during the data collection period. Learner collaboration and simplification of procedures are key to the success of the data collection phase. Another challenge encountered was the urgent requirement of high-fidelity feedback models during the training period because the limited number of models constrained the quality of the teaching. BLS training cannot proceed without handon feedback models, which are expensive. Considering massive lay learners and high demand of BLS training, the huge cost of the model hinders the flexibility and accessibility of BLS training. Substitutable models, such as personal BLS mannequins, were acceptable if the quality of BLS training was guaranteed.

One of the motivating factors of the project was the funding support from Jiangxi Provincial Department of Science and Technology from the beginning. In addition, the teaching assistants were members of Nightingale University community, which had been established in this university for more than 10 years, containing more than 1000 qualified nursing volunteers, with a culture of serving the public with their nursing knowledge and firstaid activities, which was beneficial to the success of the project.

Another motivator of this project was that the online BLS training lesson was used as an optional course for 2 years, and the lecturer was Professor Zheng of Jiangxi
Medical College, who has won the Top Ten Medical Simulation Training Center Managers and the MOOC Excellent Teacher Award of China University. One thousand one hundred forty-three students all over China received the BLS training by watching the video via mobile phone. The research team has accumulated experience of managing students and classes. University resources, such as hand-on CPR exercise models, welleducated students, teaching assistants, and the university website, were used to popularize the public mutual help notion and provide opportunities to extend training coverage and reduce cost. This project helped to shape the process of prehospital BLS and identify the changes of lay rescuer in skill and psychology.

\section{From Evidence to a Training Pathway}

Our BLS training was implemented at one of the top 100 universities in China, which is located in Southeast China. The increased rescue rate of $\mathrm{CA}$ was the trigger needed for this practice change following the Iowa Model. Once the trigger was identified, the topic was slated as a priority. The doctor was introduced to become the project manager, charged with implementing the evidencebased practice change. According to the Iowa Model, the project manager conducted a literature review to obtain the rationale for driving practice change. The research team combined the content of the best evidence, the professional judgment from emergency medicine specialist, the actual situation of universities, and students' preferences to form a BLS training program for lay undergraduates, which is shown in Figure 2. The project received ethical approval from the School of Nursing, Nanchang University, and the informed consent was obtained from all participants.

\section{Models for Change}

We used the Iowa Model of Evidence-Based Practice ${ }^{28}$ to improve training quality and Lewin's Change Theory to facilitate this practice change. The Iowa Model was initially used to summarize experience from a series of clinical practice projects, and to gradually refine and summarize specific practical steps by analyzing obstacles and enablers in the implementation of each project. ${ }^{29}$ See Figure 3 for Iowa Model implementation. Lewin summarized the organizational change process as Unfreezing-Moving-Refreezing. ${ }^{30}$ Unfreezing refers to changing the attitudes of members of an organization and encouraging them to accept new ideas. Unfreezing had begun before the implementation of the 


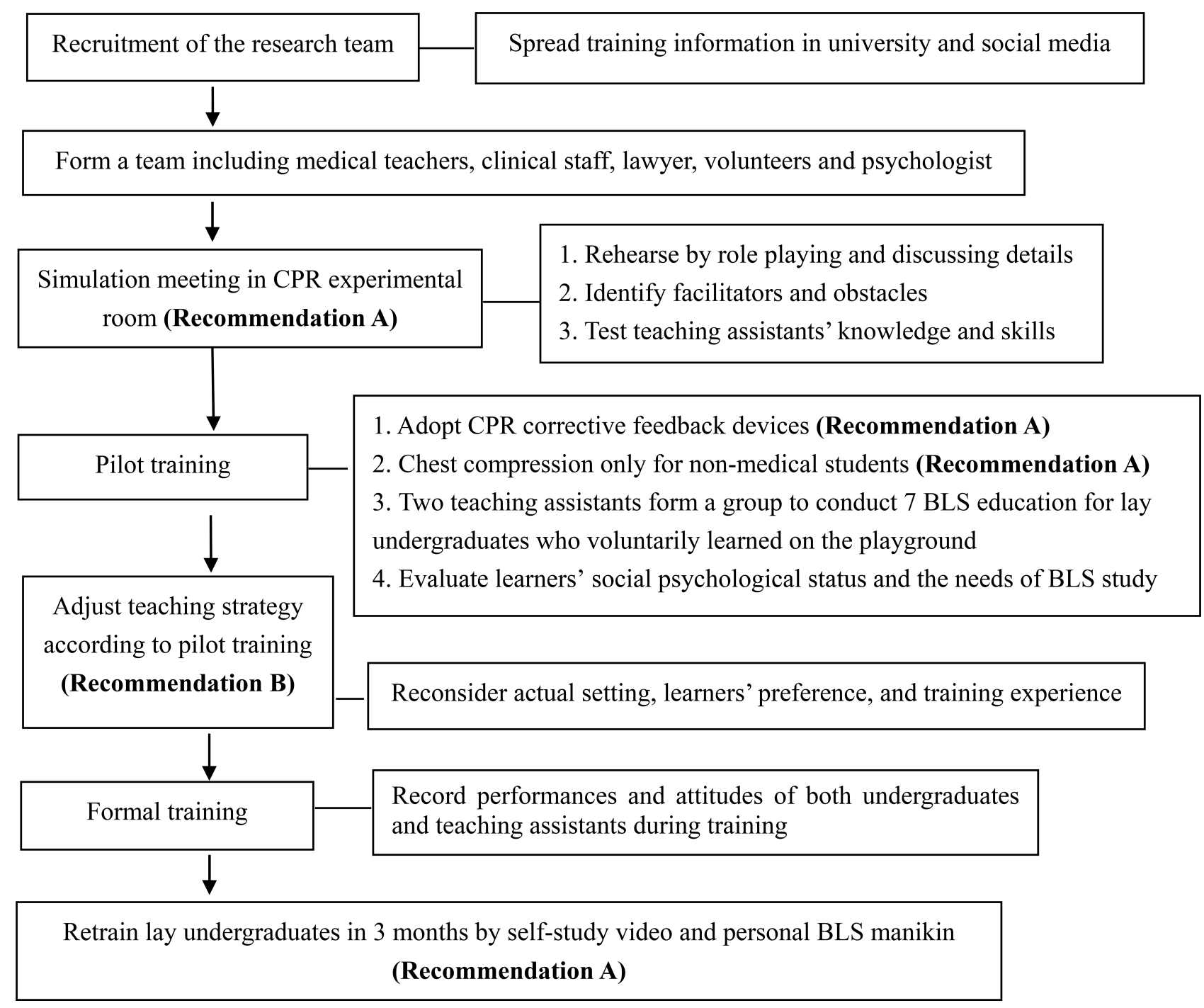

Figure 2 The evidence-based BLS training pathway for lay undergraduates.

project. We surveyed undergraduates' willingness to implement BLS and interviewed them to capture their concerns. After that, while recruiting teaching assistants, we strongly encouraged all students to join this training, and emphasized the importance of implementing prehospital BLS through social media and bulletin boards for a month to play a subtle role on students' attitude. Moving refers to the formation of new attitudes and behaviors based on acceptance of new ideas and renewed understanding. Lack of confidence and fear of failure in rescue are obstacles to their implementation of BLS. Emergency experts and teachers were already supportive of the project that was the impetus to the change. The Iowa Model steps were helpful for the moving stage of change with the lay undergraduates. Refreezing is the stage during which new behaviors are practiced and strengthened. The final stage is well underway in this university because there is a first-aid organization and volunteer association whose members will regularly participate in community activities and first-aid training propaganda under the leadership of nursing teachers and emergency experts, which is considered a routine practice on the unit.

\section{Participants and Settings}

Prehospital BLS trainings were implemented in a university ranking in the top 100 in China. Teaching assistants were recruited from 381 nursing undergraduates. We selected 10 teaching assistants from them. Inclusion criteria need to be met: 1) undergraduate, 2) having medical background or first-aid certificate, 3) having strong 


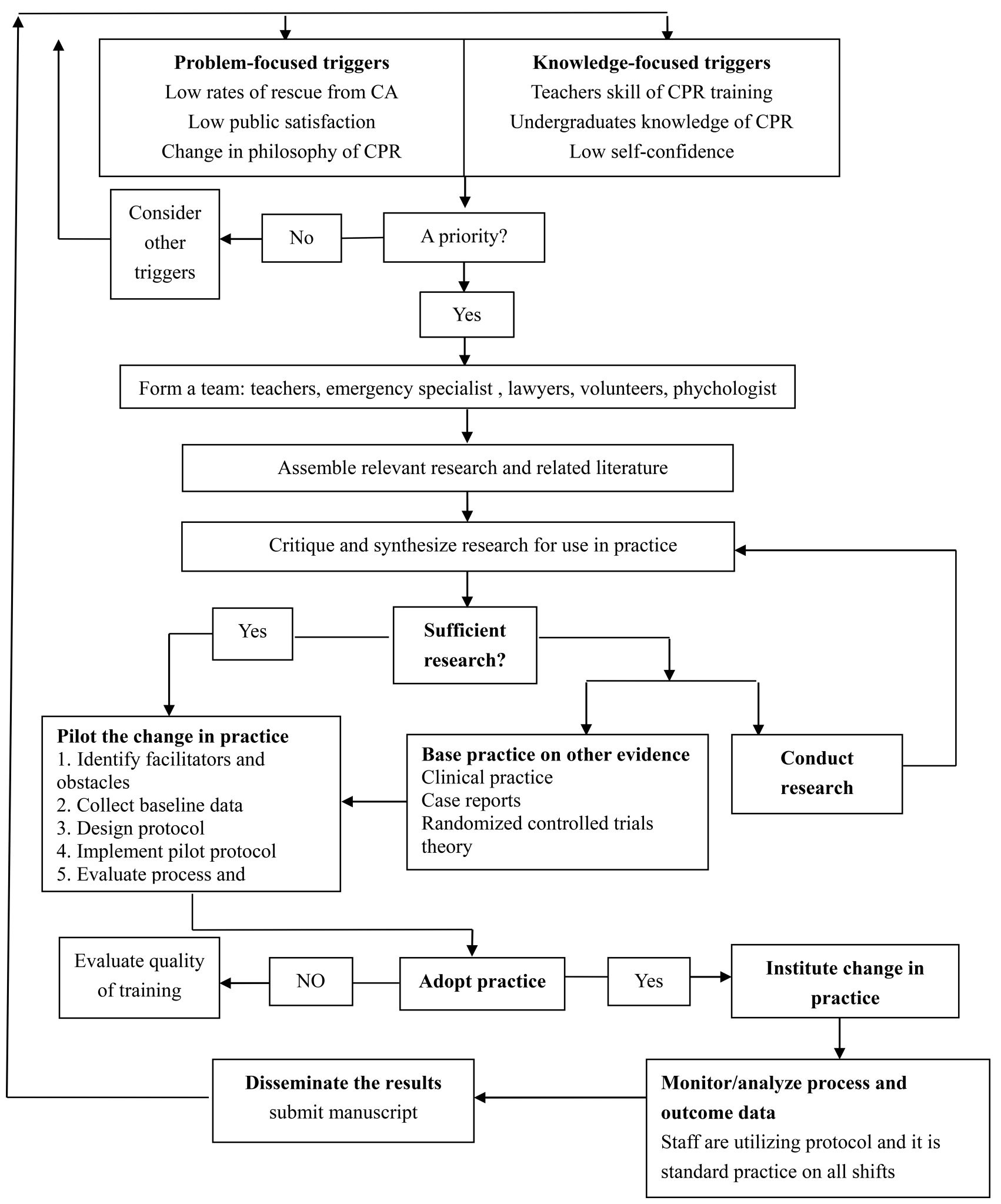

Figure 3 lowa model of evidence-based practice to implement undergraduates' BLS Training.

willingness to participate. To ensure the positive effects of peer education, we conducted a psychological evaluation and a willingness to participate survey of the
10 undergraduates. The results proved that the teaching assistants have good psychological quality and high participation enthusiasm. 
The project manager identified champion representatives from several disciplines, including an emergency doctor and nurse, two nursing teachers, a psychologist, and a lawyer during BLS training. Those representatives were charged with working as a team to communicate departmental and discipline-specific issues concerning BLS training. After compiling the issues from all of the team members, the project manager designed and led the team in a simulation experience to develop a preliminary protocol. The project manager presented evidence-based protocol.

\section{Simulation and Agreements}

Once the team members were identified, the next step was to coordinate and lead a 30 mins simulation training for teaching assistants. Prior to the simulation, the psychologist, lawyer, and emergency staff consultant verbally shared their thoughts and opinions online as they were unable to attend the actual simulation due to scheduling conflicts. Even so, their full support was conveyed to the project manager. Specifically, the lawyer explained concerns and resolutions regarding failure of resuscitation, and the psychologist voiced concerns regarding the learners' mental preparation to help build better coping strategies to avoid emotional challenges. The doctor and nurse introduced professional steps and notes on BLS. Lay undergraduates can only learn the chest compression in BLS, which can also achieve the same effect of resuscitation. The details of CPR protocol for laymen are as follows: 1) Confirm the safety of the condition, 2) Let the patient lie on the hard surface and recognize the cardiac arrest within $10 \mathrm{~s}$ (need to identify three "noes": No consciousness - no response after patting the patient's shoulders and calling him loudly; No breathing - the patient has no thoracic undulation; No heartbeat - for laymen, the necessity of checking for a pulse is no longer emphasized, but a cardiac arrest is considered whenever the patient is unconsciousness and not breathing) (3) Ask someone to call the emergency number (4) Chest compression: the compression point is the midpoint of the connection between the two nipples. The rescuer places the palm root, on the other hand, with the straight elbows. The adult should be pressed vertically with a frequency of 100-120 times/min, and a depth of 5-6cm.

The agreements reached are as follows: (1) team meeting before training was essential to rehearse by role play and discuss details, (2) two teaching assistants worked together to train lay undergraduates by hands-on corrective feedback high-fidelity simulators, (3) the proportion of teacher to assistant and student was 1:5:60, (4) training concentrated on BLS quality, (5) re-simulation training within 3 months. The teachers felt that the simulation would enhance assistants' confidence and social responsibility, and the emergency staff agreed that there would be different ways of thinking from relevant fields which were indispensable in diversity of teaching solutions.

\section{Protocol Development and Implementation}

Based on the above analysis and the agreement after simulation training, the project manager formed the evidence-based BLS training protocol by combining the best evidence, notes and observations documented during the simulation, the experts' professional judgment, and the actual needs of undergraduates. The training protocol was endorsed by all team members, and the project manager conducted multiple offline education courses for teaching assistants regarding the operating procedures and details that should be paid attention to during BLS training. All assistants took a quiz to determine whether they had a complete grasp of this protocol before they formally provided support to BLS training for lay undergraduates. The protocol was implemented with a plan to conduct 120 events of BLS training.

\section{Results \\ First Aim}

The project's first aim of developing a protocol was accomplished through the team simulations. Written notes of observations and brainstorm were taken during the simulations, and these data were used to develop the pilot protocol. The teachers guaranteed and coordinated problem-solving and good communication. The simulations were acted out in the university experimental room equipping high-fidelity CPR models. Each member participating in the simulations assumed their assigned role and role-playing being practiced until all agreed on the method and sequence of events that were ideal for this setting. The team determined to do learners' psychological preparation by understanding the protection of relative law. Emulation environment settings should be paid attention to throughout the perform, including flexible backup alternatives when CA conditions were out of control.

\section{Second Aim}

To accomplish the project's second aim, all personnel received training on the BLS protocol and completed 
a quiz and signed consent. All of them achieved a score of 100 on the educational post-test and consented to participate after the education. Not all staff members attended the faceto-face educational sessions; however, anyone assigned was required to read the protocol, complete the quiz, sign the consent, and verbalize their understanding before participating. After the education part of the project, the BLS training protocol was implemented according to the Iowa Model.

\section{Third Aim}

The third project aim of evaluation was accomplished with a survey questionnaire. According to the Iowa Model, survey responses were reviewed by the project manager with the team to ensure there is any change in this protocol after seven events occurred. The pilot protocol was trialed for the seven events of the five assisting groups, and the project manager evaluated the 35 survey responses collected from these initial events. Descriptive data were analyzed for each of the 5-Point Likert Scale survey questions using SPSS version 21. Most participants either agreed or strongly agreed on the ease of use of the protocol (Table 2). Qualitative data were summarized from the open-ended questions. Staff comments included a need for identification of scruple to perform BLS and quick reaction. This was addressed with encouragement and more communication with relative professionals in the fields of emergency, legislation, morality, reminding all teaching staff of the pilot protocol. Overall, most participants related positive responses and indicated their support regarding the ease of usage of the protocol.

Table 2 The Attitude of Teaching Assistants in 120 Events

\begin{tabular}{|l|l|l|l|}
\hline Survey Question & Number & $\begin{array}{l}\text { Mean } \\
(\text { SD) }\end{array}$ & $\begin{array}{l}\text { Median } \\
\left(\mathbf{P}_{\mathbf{2 5}}, \mathbf{P}_{\mathbf{7 5}}\right)\end{array}$ \\
\hline $\begin{array}{l}\text { In the previous } \\
\text { seven events }\end{array}$ & & & \\
$\quad$ Protocol followed & 35 & $4.19(0.23)$ & $4(4,4)$ \\
Team coordination & 35 & $4.08(0.31)$ & $4(4,4)$ \\
Comfort in role & 35 & $4.20(0.52)$ & $4(4,5)$ \\
Ease of training & 35 & $4.16(0.49)$ & $4(4,4)$ \\
\hline In the subsequent & & & \\
events & & & \\
$\quad$ Protocol followed & 85 & $4.58(0.24)$ & $5(4,5)$ \\
Team coordination & 85 & $4.78(0.27)$ & $5(5,5)$ \\
Comfort in role & 85 & $4.60(0.46)$ & $5(4,5)$ \\
Ease of training & 85 & $4.76(0.41)$ & $5(5,5)$ \\
\hline
\end{tabular}

Notes: Likert Scale scoring: 1 = strongly disagree; 2 = disagree; 3 = neutral; 4 = agree; 5 = strongly agree.
The project manager and team members reviewed the results from the first 35 BLS trainings and concluded that some adjustments were recommended at that time, including more detailed introduction before training and a specific procedure for students. Implementation continued with 85 more face-to-face training sessions. The same statistics were evaluated for each of the 4 survey questions again from the succedent surveys collected. The mean scores of each question increased with these subsequent trainings, indicating that the staff members were more comfortable in their roles and with the protocol (Table 2). In addition, we also conducted a survey on the situation of 120 learners after the first training and the re-training. The results showed that learners' BLS knowledge and skills improved significantly after the re-training. Learners' confidence, enthusiasm, satisfaction and adaptability for the evidence-based training program were also significantly enhanced (Table 3).

The open-ended question responses implied more accomplishment with the result of the training and indispensable support of the protocol as noted by specific statements made on the questionnaire, such as "the significance of teamwork" to the question about obstacles and barriers, and "go smoothly and happy to interact with the students", and "very important for learner satisfaction and mentality construction. I'm excited that we are implementing this." As the project protocol continued, assistants appeared more willing to document their opinions in detail; for example, "it is easy to communicate with the learner because we are both students", and "be proud of us teaching others life rescue technique and selfimproving in many aspects" and "the way of self-study with

Table 3 The Situation of 120 Learners After Training ( $\bar{x} \pm s)$

\begin{tabular}{|l|l|l|l|l|}
\hline $\begin{array}{l}\text { Survey } \\
\text { Question }\end{array}$ & $\begin{array}{l}\text { Training for } \\
\text { the First } \\
\text { Time }\end{array}$ & $\begin{array}{l}\text { Re-Training } \\
\text { After 3 } \\
\text { Months }\end{array}$ & $\mathbf{t}$ & $\mathbf{P}$ \\
\hline $\begin{array}{l}\text { Objective } \\
\text { assessment } \\
\text { Knowledge }\end{array}$ & $4.61 \pm 1.547$ & $5.81 \pm 1.925$ & 3.921 & $0.000 \mathrm{I}$ \\
Skills & $4.88 \pm 1.771$ & $5.90 \pm 1.864$ & 3.212 & 0.002 \\
\hline Subjective & & & & \\
assessment & & & & \\
Confidence & $15.87 \pm 3.256$ & $20.73 \pm 3.404$ & 8.327 & 0.0001 \\
Satisfaction & $15.30 \pm 2.553$ & $17.63 \pm 3.357$ & 4.446 & 0.0016 \\
Enthusiasm & $10.42 \pm 2.097$ & $12.48 \pm 2.693$ & 4.878 & 0.0003 \\
Adaptability & $10.15 \pm 2.009$ & $11.86 \pm 2.699$ & 4.072 & 0.0007 \\
\hline
\end{tabular}

Notes: The full score of BLS knowledge and skills assessment is 10 points respectively. Likert Scale scoring: 1 = strongly disagree; 2 = disagree; 3 = neutral; 4 = agree; 5 = strongly agree. 
video to learn BLS is common but inefficient, on the other hand, calling for a more practical method for popularity". This provided the impetus for the effective BLS teaching strategy in accordance with actual Chinese condition.

In the first phase of implementation, responses to the open-ended questions revealed that psychological scruples of being dunned for hurting victim without intention and lack of confidence. This issue seemed to be resolved during the second phase of pilot implementation by the explanation of experts.

After the project, the following minor amendments were made to the pilot protocol: (1) assign a professional and several teaching assistants to manage online questions on social media, which will be beneficial to trainings as supplementary (2) ensure that high-fidelity models are easily accessible for the students (3) to solve communication issues, regular meetings will be held to exchange experiences and opinions. No major procedural changes of the pilot protocol were warranted according to the survey results.

\section{Discussion}

BLS is a world-wide accepted prehospital first aid when CA occurs, which is a required step of CPR. ${ }^{13}$ Meeting the needs of the public mutual help, raising satisfaction, and enhancing security for people, especially those experiencing coronary heart disease and other chronic illnesses make highlight the benefits of this evidence-based practice change. The protocol was tested among 120 learners and proven to be feasible in university.

Because of this training change, the role of the lay rescuer and process was more clearly defined to provide first eyewitness BLS at the site of the emergency. The psychological consultant and lawyer were absent from actual training activities but took part in the protocol amendment through distant online meetings. The teaching assistant was proven to play a key role in the success of this project and continues to lead this training change. When the project was complete, the team reported that BLS training in scheduled protocol had become peer-study -driven and was considered routine trainings.

In this project, members from each key discipline came together and collectively worked through all concerns and issues. The simulation allowed for variation of the opinions and each team members' voice to be heard. In the process of implementation, each participant was able to adjust the proposed change on their individual role and responsibility for BLS training. The team communicated and demonstrated to staff in their respective aspects that BLS training could be successful so that lay undergraduates could contribute to prehospital first aid and the spread of a public spirit of dedication all over Chinese society.

For future projects such as this one, we recommend providing more face-to-face clear-up sessions and reaching more health-care professionals and enhancing self-education with better organized social media. Recommended next steps include implementing individual BLS training in less than the 20 mins currently outlined in the protocol, eventually increasing students' retrain frequency, as well as activation, confidence, and acceptance. Expanding opportunities of BLS theme activities for trained students to enhance the study effects and awareness of public BLS. In addition, taping each learner 's study video was a necessary supportive option to review and share on their network and circle of friends to increase attention. In addition, mass media and government efforts should be made for dissemination and financial support.

Future research should evaluate different aptitudes and education background of learners before education to modify training strategies. Another area of future study includes developing feedback models for more flexible states and lower cost with multi-disciplinary and multimajor resources, which could be affordable for normal Chinese families or government paid because they would potentially boost the popularity of BLS and supplement professional urgent care.

\section{Conclusion}

The purpose of this study is to explore a convenient BLS skills popularization model suitable for Chinese national conditions. The evidence-based concept is recommended to address concerns and issues of public service teaching staff, which can decrease anxiety and misunderstanding to prehospital emergency field. In this project, we used nursing undergraduates as peer instructors to provide free BLS training for lay undergraduates. The team members from each key discipline came together and collectively worked to solve all concerns and issues related to implementation of lay undergraduates' BLS training. This simulation allows the voice of each member to be heard. By improving the quality of BLS training, the development and implementation of evidence-based protocol could elevate the lay undergraduates' BLS knowledge, skills and confidence, satisfaction, enthusiasm and adaptability, and be considered by peer instructors to be convenient and easy to use, 
indicating that this BLS training protocol for lay undergraduates in universities can be implemented smoothly.

This study showed that BLS training for lay undergraduates is beneficial and efficient, desired by society, and considered an acceptable practice in multidisciplinary health care and training teams. In this way, we hope that while improving the first-aid skills of undergraduates, they can spread BLS knowledge and skills to residents and expand their influence in the communities, by which a network of community first-aid involving laymen is gradually established.

\section{Acknowledgments}

We would like to thank our researchers for their hard work and reviewers for their valuable advice.

\section{Funding}

This study was financially supported by Natural Science Foundation of Jiangxi Province (20171BAB215027) and 2019 Nanchang University Research and Training Program.

\section{Disclosure}

All authors declare that they have no conflicts of interest in this work.

\section{References}

1. Panchal AR, Cash RE, Crowe RP, et al. Delphi analysis of science gaps in the 2015 American Heart Association Cardiac Arrest Guidelines. J Am Heart Assoc. 2018;7(13):8571. doi:10.1161/ JAHA.118.008571

2. Kose S, Akin S, Mendi O, Goktas S. The effectiveness of basic life support training on nursing students' knowledge and basic life support practices: a non-randomized quasi-experimental study. Afr Health Sci. 2019;19(2):2252. doi:10.4314/ahs.v19i2.51

3. González-Salvado V, Abelairas-Gómez C, Peña-Gil C, et al. Basic life support training into cardiac rehabilitation programs: a chance to give back. A community intervention controlled manikin study. Resuscitation. 2019;19(2):14-20.

4. Nishi T, Takei Y, Kamikura $T$, Oht $\mathrm{K}$, Hashimoto $\mathrm{M}$, Inaba $\mathrm{H}$. Improper bystander-performed basic life support in cardiac arrests managed with public automated external defibrillators. Am J Emerg Med. 2015;33(1):43-49. doi:10.1016/j.ajem.2014.10.018

5. Wang LX. Development strategy on cardiopulmonary resuscitation in China. Chin Crit Care Med. 2015;27(3):161-163.

6. American Heart Association (AHA). Part 1: executive Summary: 2015 American Heart Association Guidelines Update for Cardiopulmonary Resuscitation and Emergency Cardiovascular Care. Circulation. 2015;132(18):315-367. doi:10.1161/CIR.0000000000000252

7. Cardiopulmonary Resuscitation Specialized Committee of Chinese Research Hospital Association(CRSC). 2016 National consensus on cardiopulmonary resuscitation in China. Chin J Disaster Med. 2017;5 (1):1-23.

8. Leong BSH. Bystander CPR and survival. Singapore Med J. 2011;52 (8):573-575.
9. Pan J, Lu YQ. Discussion on establishment of network training and emergency community framework for cardiopulmonary resuscitation in China. Chin J Crit Care Med. 2012;5(1):25.

10. Jarrah S, Judeh M, AbuRuz ME. Evaluation of public awareness, knowledge and attitudes towards basic life support: a cross-sectional study. BMC Med Educ. 2018;18(1):1-7. doi:10.1186/s12909-017$1038-5$

11. de Ruijter PA, Biersteker HA, Biert J, van Goor H, Tan EC. Retention of first aid and basic life support skills in undergraduate medical students. Med Educ Online. 2014;19(1):24841. doi:10.3402/meo. v19.24841

12. Galbraith K, Ward A, Heneghan C. A real-world approach to Evidence-Based Medicine in general practice: a competency framework derived from a systematic review and Delphi process. $B M C$ Med Educ. 2017;17(1):78. doi:10.1186/s12909-017-0916-1

13. American Heart Association (AHA). American Heart Association guidelines update for cardiopulmonary resuscitation and emergency cardiovascular care. Circulation. 2018;137:7-13. doi:10.1161/ CIRCULATIONAHA.117.029932

14. International Liaision Committee on Resuscitation(ILCOR). Part 1: executive summary: 2015 International Consensus on Cardiopulmonary Resuscitation and Emergency Cardiovascular Care Science with Treatment Recommendations. Circulation. 2015;132:S2-S39. doi:10. 1161/CIR.0000000000000270

15. International Liaision Committee on Resuscitation(ILCOR). 2017 International consensus on cardiopulmonary resuscitation and emergency cardiovascular care science with treatment recommendations summary. Circulation. 2017;136(23). doi:10.1161/CIRCULATIONAHA.117.027 612

16. Pan LL, Zhao AP, Qi Q. Systematic evaluation of training effect of high-fidelity simulator (HFS) in medical training of cardiopulmonary resuscitation. Chin J Pract Nurs. 2014;030(17):60-64.

17. Sahu S, Lata I. Simulation in resuscitation teaching and training, an evidence based practice review. J Emerg Trauma Shock. 2010;3 (4):378-384. doi:10.4103/0974-2700.70758

18. Lyon RM, Clarke S, Milligan D, Clegg GR. Resuscitation feedback and targeted education improves quality of pre-hospital resuscitation in Scotland. Resuscitation. 2012;83(1):0-75. doi:10.1016/j. resuscitation.2011.07.016

19. Perkins GD, Lall R, Quinn T, et al. Mechanical versus manual chest compression for out-of-hospital cardiac arrest: a pragmatic, cluster randomized controlled trial. Lancet. 2015;385(9972):947-955. doi:10.1016/S0140-6736(14)61886-9

20. Isbye DL, Rasmussen LS, Lippert FK, Rudolph SF, Ringsted CV. Laypersons may learn basic life support in $24 \mathrm{~min}$ using a personal resuscitation manikin. Resuscitation. 2006;69(3):435-442. doi:10. 1016/j.resuscitation.2005.10.027

21. Lu YQ, Pan J, Zhang XP. Study on related influencing factors of cardiopulmonary resuscitation training in Community. Chin J Crit Care Med. 2013;6(2):1-2.

22. Daniels SR. CPR training in schools. J Pediatr. 2017;181:1. doi:10.1016/j.jpeds.2016.12.008

23. Cho GC, Sohn YD, Kang KH, et al. The effect of basic life support education on laypersons willingness in performing bystander hands only cardiopulmonary resuscitation. Resuscitation. 2010;81 (6):691-694. doi:10.1016/j.resuscitation.2010.02.021

24. Blewer AL, Leary M, Esposito EC, et al. Continuous chest compression cardiopulmonary resuscitation training promotes rescuer self-confidence and increased secondary training: a hospital-based randomized controlled trial. Crit Care Med. 2012;40(3):787-792. doi:10.1097/CCM.0b013e318236f2ca

25. Bogle B, Mehrotra S, Chiampas G, Aldeen AZ. Assessment of knowledge and attitudes regarding automated external defibrillators and cardiopulmonary resuscitation among American University students. Emerg Med J. 2013;30(10):837-841. doi:10.1136/ emermed-2012-201555 
26. Cave DM, Aufderheide TP, Beeson J, et al. Importance and implementation of training in cardiopulmonary resuscitation and automated external defibrillation in schools: a science advisory from the American Heart Association. Circulation. 2011;123(6):691-706. doi:10.1161/CIR.0b013e31820b5328

27. Aaberg AM, Larsen CE, Rasmussen BS, Hansen CM, Larsen JM. Basic life support knowledge,self-reported skills and fears in Danish high school students and effect of a single 45-min training session run by junior doctors; a prospective cohort study. Scand J Trauma Resusc Emerg Med. 2014;22(1):1-6. doi:10.1186/1757-7241-22-24
28. Titler MG, Kleiber C, Steelman V, et al. Infusing research into practice to promote quality care. Nurs Res. 1994;43(5):307. doi:10.1097/00006199-199409000-00009

29. Titler MG, Kleiber C, Steelman VJ, Rakel BA, Goode CJ. The Iowa model of evidence-based practice to promote quality care. Crit Care Nurs Clin North Am. 2002;13(4):497-509. doi:10.1016/S08995885(18)30017-0

30. Beck SJ. Review of Field theory in social science: selected theoretical papers. Am J Orthopsychiatry. 1953;23(1):213-214. doi:10.1037/ $\mathrm{h} 0096603$

\section{Publish your work in this journal}

Risk Management and Healthcare Policy is an international, peerreviewed, open access journal focusing on all aspects of public health, policy, and preventative measures to promote good health and improve morbidity and mortality in the population. The journal welcomes submitted papers covering original research, basic science, clinical \& epidemiological studies, reviews and evaluations, guidelines, expert opinion and commentary, case reports and extended reports. The manuscript management system is completely online and includes a very quick and fair peer-review system, which is all easy to use. Visit http://www.dovepress.com/testimonials.php to read real quotes from published authors. 